\title{
Intelligent Intra-Field Interpolation for Motion Compensated Deinterlacing
}

\author{
Yu-Cheng Fan \\ Department of Electrical Engineering \\ National Taiwan University \\ Taiwan, R.O.C. \\ d9921004@ee.ntu.edu.tw \\ Hen-Wai Tsao \\ Department of Electrical Engineering \\ National Taiwan University \\ Taiwan, R.O.C. \\ tsaohw@cc.ee.ntu.edu.tw
}

\author{
Hung-Shih Lin \\ Department of Electrical Engineering \\ National Taiwan University \\ Taiwan, R.O.C. \\ hozlin@twt1.com.tw \\ Ching-Chih Kuo \\ Department of Electrical Engineering \\ Hwa Hsia Institute of Technology \\ Taiwan, R.O.C. \\ dean@cc.hwh.edu.tw
}

\begin{abstract}
This paper proposes a novel intelligent intra-field interpolation for motion compensated deinterlacing. This method combines the advantage of cubic curve fitting interpolation and fuzzy edge interpolation to overcome resolution degradation caused by incorrect motion vector. The intelligent intra-field interpolation scheme alleviates resolution degradation in areas where object motion cannot be well tracked by motion estimation and produces deinterlaced pictures with better visual quality, less flicker, and imperceptible artifacts. Experimental results show that the developed method can indeed generate high quality intra-interpolation picture for motion areas.
\end{abstract}

Index Terms - Cubic curve fitting interpolation, deinterlacing, fuxzy edge interpolation, motion compensation.

\section{INTRODUCTION}

$\mathrm{R}$ ecent advances of high-definition television (HDTV) and multimedia PC strongly support the interoperability between interlaced and progressive videos. Deinterlacing is an important process for converting a video sequence from interlaced scan into progressive scan to be displayed on progressive devices. The input video field, which contains samples from either odd or even lines of an image, has to be converted to a frame. This frame represents the same image as the corresponding input field but contains the samples from all the lines [1]-[4].

However, since the sampling process of interlaced TV signals along the vertical direction does not satisfy the Nyquist sampling theorem, the linear sampling-rate conversion theory cannot be applied for effective interpolation [2]. In order to solve deinterlacing problems, motion compensated deinterlacing provides a solution to this problem by interpolating along the motion trajectory. The motion compensated algorithm allows us to virtually convert a moving sequence into a stationary one. Not all temporal information changes can be adequately described with motion vectors. Scene changes, fades, and concealed or obscured backgrounds, are several examples of difficulties encountered. As a consequence, the key action of the motion compensated deinterlacing algorithm is to obtain a motion vector equivalent to the true motion and an error detection to estimate the correctness of the motion vector.

$\mathrm{Li}$ proposed a motion detection/compensation between opposite parity fields in interlaced video sequences [5]. Kwon presented directional interpolation and motion compensation for deinterlacing [6]. Ryu adopted local spectra analysis based motion compensated deinterlacing algorithm [7]. Patti developed an approach for motion compensated, reduced-order model Kalman filtering for restoration of progressive and interlaced video [8].

However, when the motion vector is judged to be incorrect for some interpolated pixel, intra-field interpolation will bring about resolution degradation. In order to alleviate resolution degradation in the area where object motion cannot be tracked well by motion estimation, this paper presents an intelligent intra-field interpolation for motion compensated deinterlacing scheme to improve both the one and two dimensional intra-field interpolation performances. This method combines the advantage of cubic curve fitting interpolation and fuzzy edge interpolation to overcome resolution degradation and produce deinterlaced pictures with better visual quality, less flicker, and imperceptible artifacts.

The paper is organized as follows. The cubic curve fitting interpolation is described in Section II. Fuzzy edge interpolation is presented in Section III. In Section IV, the intelligent intra field interpolation rule is stated. In Section V, the experimental results are shown. The conclusion of this paper is given in Section VI. 


\section{CubIC CuRve FITTING INTERPOLATION}

A simple vertical interpolation called line averaging uses only two pixels, those above and below $P_{i}(x, y, n)$, respectively. Cubic curve fitting interpolation uses four pixels at the vertical neighborhood of $P_{i}(x, y, n)$ to obtain better interpolation results. Consider the transition of luminance in the $y$ direction approximated as a third order function of $y$ (see Figure 1):

$$
L(y)=a+b y+c y^{2}+d y^{3}
$$

Suppose $L(0)$ is the pixel to be interpolated and $L(-3), L(-1)$, $L(1)$ and $L(3)$ correspond to four sample pixels of the original field, respectively. With $\mathrm{L}(-3), \mathrm{L}(-1), \mathrm{L}(1)$ and $\mathrm{L}(3)$ already known, the following four equations can be obtained from (1):

$$
\begin{aligned}
& L(-3)=a-3 b+9 c-27 d \\
& L(-1)=a-b+c-d \\
& L(1)=a-b+9 c+d \\
& L(3)=a+3 b+9 c+27 d
\end{aligned}
$$

Solving (2), the interpolated pixel can be written as:

$$
\begin{aligned}
& L(0)=\left[\begin{array}{llll}
1 & y & y^{2} & y^{3}
\end{array}\left[\begin{array}{l}
a \\
b \\
c \\
d
\end{array}\right]_{y=0}\right. \\
& =\left[\begin{array}{llll}
1 & 0 & 0 & 0
\end{array}\left[\begin{array}{cccc}
1 & -3 & 9 & -27 \\
1 & -1 & 1 & -1 \\
1 & 1 & 1 & 1 \\
1 & 3 & 9 & 27
\end{array}\right]^{-1}\left[\begin{array}{c}
L(-3) \\
L(-1) \\
L(1) \\
L(3)
\end{array}\right]\right. \\
& =\left[\begin{array}{llll}
-0.0625 & 0.5625 & 0.5625 & -0.0625 \\
L(-1) \\
L(1) \\
L(3)
\end{array}\right]
\end{aligned}
$$

Finally, the result of cubic curve fitting interpolation is:

$$
\begin{aligned}
P_{\text {cubic }}(x, y, n) & =-0.0625 P_{0}(x, y-3, n)+0.5625 P_{0}(x, y-1, n) \\
& +0.5625 P_{0}(x, y+1, n)-0.0625 P_{0}(x, y+3, n)
\end{aligned}
$$

\section{FUZZY EDGE INTERPOLATION}

Edge-dependent interpolation is an important method for improving the resolution of intra-field interpolation, especially on moving diagonal lines. There have been many edge-dependent interpolation methods proposed before [9]-[11] However, artifacts still occur in edge-dependent interpolation when edge detection fails, especially at thin edges and non-dominant edges (irregular regions). At thin edges, the detected edge often has a direction opposite to that of the true edge. At non-dominant edges, pixels have very little correlation with one another. In order to avoid erroneous detection of edges, a method for fuzzy edge detection has been proposed in [10]. In this paper, we further improve this fuzzy edge detection by enlarging the detection angles and successfully reduce the artifacts due to erroneous edge detection. A fuzzy detector follows the same processing steps as a fuzzy controller, i.e. inference of fuzzification, rule and defuzzification. Figure 2(a) shows the processing window of the current field considered by the fuzzy edge detector. Seven directions are utilized to interpolate the missing pixel $P_{i}(x, y, n)$. Pixel differences used as inputs to the fuzzy detector are defined as:

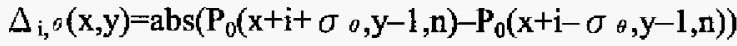

where $\mathrm{i}$ is the horizontal offset of $\mathrm{x}, \theta$ is the edge direction among $\left\{23^{\circ}, 31^{\circ}, 45^{\circ}, 90^{\circ},-45^{\circ},-31^{\circ},-23^{\circ}\right\}$, and $\sigma_{\theta}$ is the orientation offset of $x$ among $\{-3,-2,-1,0,1,2,3\}$, respectively, corresponding to $\theta$ values listed above. The inputs are converted into fuzzy membership values by the pre-defined membership function, as shown in Figure 2(b). The membership function named $s m a l l_{ \pm \theta}$ is identical for directions $\theta$ and $-\theta$ to perform symmetrical evaluation. From Figure $2(\mathrm{~b})$, small ${ }_{ \pm \theta}$ has two parameters: $a_{ \pm \theta}$ and $d_{ \pm \theta}$. If $\Delta_{\mathrm{i}, \theta}(\mathrm{x}, \mathrm{y})$ is smaller than $d_{ \pm \theta}$ and larger than $a_{ \pm \theta}$, the grade of membership (membership value) $\mu_{\theta}$ decreases. If $\Delta_{\mathrm{i}, \theta}(\mathrm{x}, \mathrm{y})$ is smaller than $a_{ \pm \theta}$, it is considered to have a truth level $\mu_{\theta}$ equal to one. Fuzzy rules for the fuzzy detector characterize the control policy needed to infer fuzzy control decisions. A rule is described as a conditional statement in which the antecedents are the conditions and the consequence is the decision. The fuzzy rule used in this paper is shown in TABLE I. For example, the rule of angle $45^{\circ}$ is stated as:

$$
\begin{aligned}
& \text { if } \Delta_{-1,45^{*}}, \Delta_{0,45^{*}}, \Delta_{1,45}, \in \text { SMALL }_{ \pm 45} . \\
& \text { then set } \text { s.5 }_{45}=1
\end{aligned}
$$

To make the final decision of edge direction, defuzzification for the fuzzy detector takes the direction with the maximum membership value as:

$$
\operatorname{direction}(\mathrm{x}, \mathrm{y})=\max _{\theta}\left(\mu \theta \mid \operatorname{set}_{\theta}=1\right)
$$

If several equal maximum values are obtained, the following priority order will be used to make the final decision: $90^{\circ},-45^{\circ}$, $45^{\circ},-31^{\circ}, 31^{\circ},-23^{\circ}, 23^{\circ}$. Futhermore, the membership parameters are selected as $\mathrm{a}_{0}{ }^{\circ}>\mathrm{a}_{ \pm 45^{\circ}}>\mathrm{a}_{ \pm 31}{ }^{\circ}>\mathrm{a}_{ \pm 23}{ }^{\circ}$ and $\mathrm{d}_{0}{ }^{\circ}>\mathrm{d}$ ${ }_{ \pm 45^{\circ}}{ }^{\circ}>\mathrm{d}_{ \pm 31}{ }^{\circ}>\mathrm{d}_{ \pm 23}{ }^{\circ}$ rather than all identical to achieve a better detection. This results in a bias toward large angles since smaller angles should cover a wider range and need a more critical detection rule. Finally, the result of fuzzy edge interpolation is:

$\mathbf{P}_{\text {fzedge }}(\mathrm{x}, \mathrm{y}, \mathrm{n})=0.5 \cdot \mathbf{P}_{0}(\mathrm{x}+\sigma \theta, \mathrm{y}-1, \mathrm{n})+0.5 \cdot \mathrm{P}_{0}(\mathrm{x}-\sigma o, \mathrm{y}+1, \mathrm{n})$

\section{INTRA FIELD INTERPOLATION RULE}

The final results of intra-field interpolation in our design can be obtained from the following rules: 


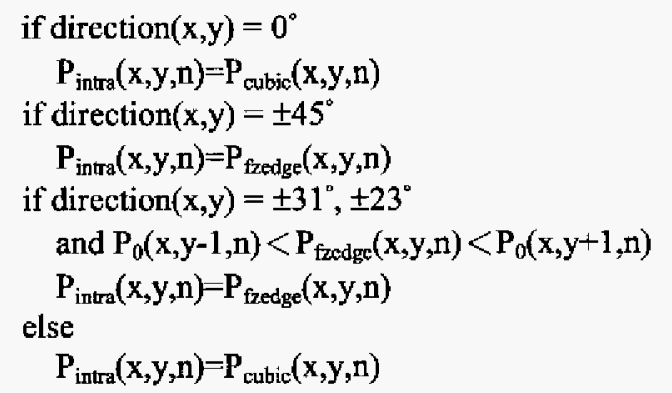

The rule $P_{0}(x, y-1, n)<P_{f_{z e d g e}}(x, y, n)<P_{0}(x, y+1, n)$ is used to further reduce artifacts occurring in edge-dependent interpolation, especially for lower angles in moving regions.

\section{EXPERIMENTAL RESULTS}

This section presents simulation results and the associated analyses of the proposed motion compensated deinterlacing method.

Figure 3(a) and Figure 3(c) shows two different parts in a picture from weather sequence that are deinterlaced by vertical interpolation. Figure 3(b) and Figure 3(d) shows the same parts in the same picture that are deinterlaced by the proposed intra-field interpolation. It can be seen that the proposed intra-field interpolation obtains smoother dominant edge in Figure 3(b) and introduces less error at thin edge in Figure 3(d).

Video quality is still a subjective matter. It is difficult to design a fully reliable objective performance criterion although the MSE is frequently used as such in the literature. It requires progressively scanned original sequences for comparison with interlaced sequences. In this paper, both MSE measurement and subjective view evaluation are used. Motion compensated deinterlacing is applied to re-conversion, and the re-converted pictures are compared with the original $352 \times 288$ progressive ones through the quantities of MSE and PSNR.

Quantitatively, the PSNRs of our deinterlacing scheme for ten CIF sequences are compared with those of several other methods, shown in Figure 4. Obviously, our method achieves better results of PSNR performance than the other methods, even by $10 \mathrm{~dB}$ in an extreme case. The three test sequences of $352 \times 288$ progressive pictures are shown in Figure 5(a)-(c). The MC-deinterlaced pictures are shown in Figure 5(d)-(f), respectively. Qualitatively, it can be seen from the figures that high quality and artifact-free results are obtained in these four video sequences.

\section{CONCLUSION}

A new intelligent intra-field interpolation for motion compensated deinterlacing is developed in this paper. The intelligent interpolation rule is used to further reducc artifacts occurring in edge-dependent interpolation, especially for lower angles in moving regions. This method employs cubic curve fitting interpolation and fuzzy edge interpolation to alleviate resolution degradation. The proposed algorithm was tested on several video sequences. Experimental results show that the developed method do overcome resolution degradation caused by incorrect motion vector and provides better video quality.

\section{REFERENCES}

[1] K. Jack, Video Demystified - A Handbook for the Digital Engineer, $3^{\text {rd }}$, LLH Technology Publishing, VA, 2001.

[2] E. B. Bellers and G. de Haan, De-interlacing - A Key Technology for Scan Rate Conversion, Elsevier, 2000.

[3] G. De Haan, E. B. Bellers, "Deinterlacing-an overview," Proceedings of the IEEE, vol. 86, pp.1839-1857, Sept, 1998.

[4] H. Y. Lee, T. M. Bae, J. W. Park, K. W. Song, and Y. H. Ha, "Efficient scan conversion with edge enhancement and its VLSI architecture," Proceedings of the IEEE Region 10 Conference, TENCON 99, vol. 1, pp: 131-134, Sept. 1999.

[5] R Li, B. Zheng, and M. L. Liou, "Reliable motion detection/compensation for interlaced sequences and its applications to deinterlacing," IEEE Transactions on Circuits and Systems for Video Technology, vol. 10, no. 1, pp: 23-29, Feb. 2000.

[6] O. Kwon, K. Sohn, and C. Lee, "Deinterlacing using directional interpolation and motion compensation," IEEE Transactions on Consumer Electronics, vol. 49, no. 1, pp: 198-203, Feb. 2003.

[7] C. Ryu, S. P. and Kim, "Deinterlacing using motion compensated local spectra" Twenty-Ninth Asilomar Conference on Signals, Systems and Computers, vol. 2, pp: 1394-1397, Oct. 1995.

[8] A. J. Patti, A. M. Tekalp, and M. I. Sezan, "A new motion-compensated reduced-order model Kalman filter for space-varying restoration of progressive and interlaced video," IEEE Transactions on Image Processing, vol. 7, no. 4, pp. 543-554, April 1998.

[9] H. Y. Lee, T. M. Bae, J. W. Park, K. W. Song, and Y. H. Ha, "Efficient scan conversion with edge enhancement and its VLSI architecture," Proceedings of the IEEE Region 10 Conference, TENCON 99, vol. 1, pp: 131-134, Sept. 1999

[10] F. Michaud, C. T. Le Dinh, and G. Lachiver, "Fuzzy detection of edge-direction for video line doubling," IEEE Trans. on Circuits and Systems for Video technology, vol. 7, no. 3, pp. 539-542, June 1997.

[11] C. J. Kuo, C. Liao, and C. C. Lin, "Adaptive interpolation technique for scanning rate conversion," IEEE Trans. on Circuits and Systerts for Video technology, vol. 6, no. 3, pp: 317-321, April 1996.

TABLE I

RULES FOR FUZZY EDGE DETECTION

\begin{tabular}{|c|c|c|}
\hline Inputs for $P_{i}(x, y, n)$ & Fuxzy Sets & $\operatorname{Set}_{\theta}=1$ \\
\hline$\Delta_{0,90^{\circ}}$ & $\operatorname{small}_{ \pm 0} 0^{\circ}$ & $0^{\circ}$ \\
\hline$\Delta_{-1,-45^{\circ}} \quad \Delta_{0,-45^{\circ}} \quad \Delta_{1,-45^{\circ}}$ & $\operatorname{small}_{ \pm 45^{\prime}}$ & $-45^{\circ}$ \\
\hline$\Delta_{-1,45^{\circ}} \quad \Delta_{0,45^{\circ}} \quad \Delta_{1,45^{\circ}}$ & $\operatorname{small}_{\perp 45^{\prime}}$ & $45^{\mathrm{n}}$ \\
\hline$\Delta_{-2,-31^{*}} \Delta_{-1,-31^{*}} \Delta_{0,-31^{\prime}} \Delta_{1,-31^{*}} \Delta_{2,-31^{\circ}}$ & small $_{ \pm 31^{\circ}}$ & $-31^{\circ}$ \\
\hline$\Delta_{-2,31^{*}} \Delta_{-1,31^{*}} \Delta_{0,31^{*}} \Delta_{1,31^{*}} \Delta_{2,31^{*}}$ & small $_{ \pm 31^{\circ}}$ & $31^{\circ}$ \\
\hline$\Delta_{-2,-23^{*}} \Delta_{-1,-23^{*}} \Delta_{0,-23^{*}} \Delta_{1,-23^{*}} \Delta_{2,-23^{*}}$ & small $_{ \pm 23^{*}}$ & $-23^{\circ}$ \\
\hline$\Delta_{-2,23^{*}} \Delta_{-1,23^{*}} \Delta_{0,23^{*}} \Delta_{1,23^{*}} \Delta_{2,23^{*}}$ & small $_{ \pm 23^{*}}$ & $23^{\circ}$ \\
\hline
\end{tabular}




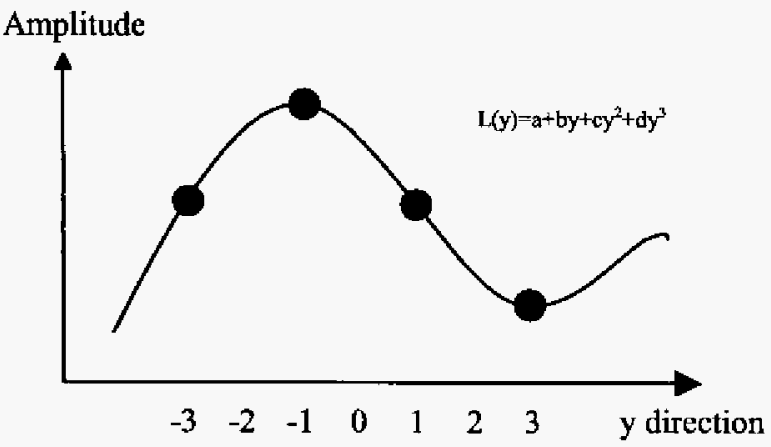

Figure 1. Luminance transition in y direction

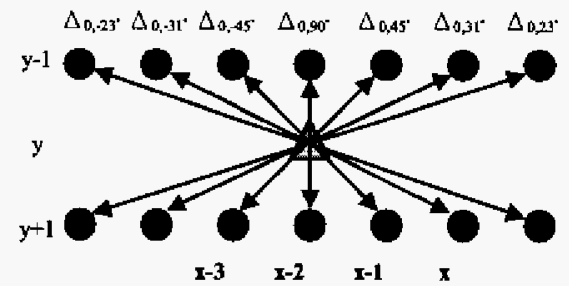

(a)

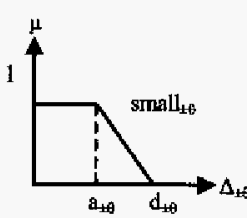

member function

(b)

Figure 2. Fuzzy edge interpolation: (a) processing window (b) member function.

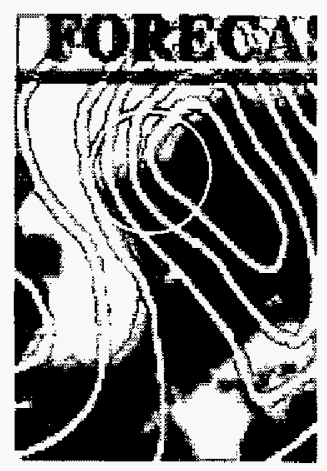

(a)

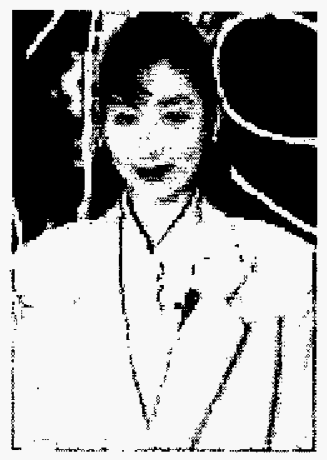

(c)

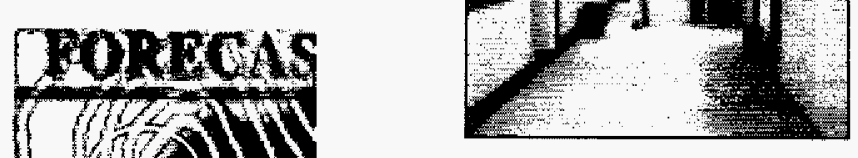

(a)

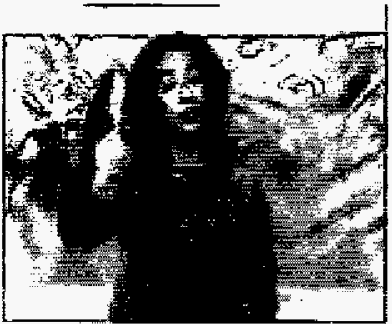

(b)

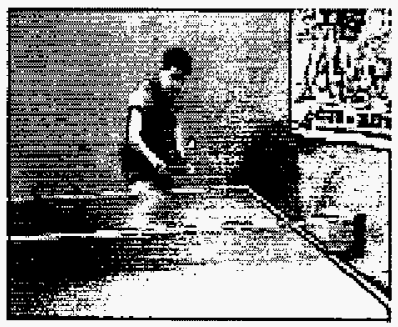

(c)

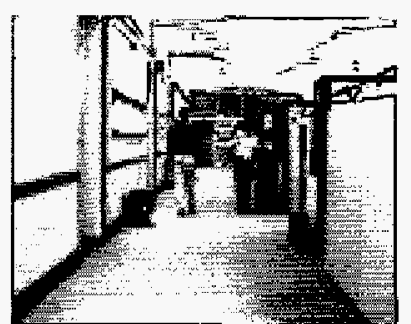

(d)

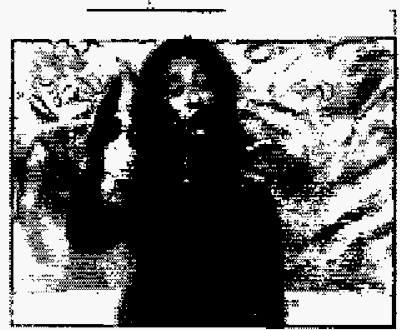

(c)

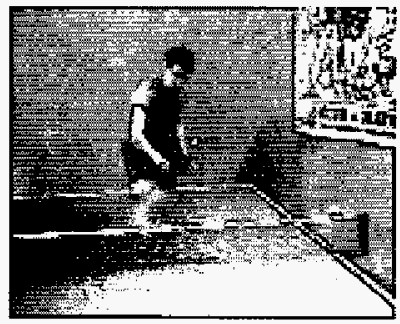

(f)

Figure 3. Results of intra-field interpolation (a) weather: vertical interpolation. (b) weather: proposed interpolation, (c) weather: vertical interpolation, (d) weather: proposed interpolation.

Figure 5. $352 \times 288$ originally progressive sequences: (a) "Hall Monitor," (b) "Silence," (c) "Table." $352 \times 288$ deinterlacing results: (d) "Hall Monitor," (e) "Silence," (f) "Table." 